ARTICLE

https://doi.org/10.1038/s41467-018-07831-5

\title{
Size-dependent kinetics during non-equilibrium lithiation of nano-sized zinc ferrite
}

Jing Li ${ }^{1}$, Qingping Meng ${ }^{2}$, Yiman Zhang ${ }^{3}$, Lele Peng ${ }^{4}$, Guihua Yu (D) ${ }^{4}$, Amy C. Marschilok ${ }^{1,3,5}$, Lijun Wu (D) ${ }^{2}$, Dong Su ${ }^{6}{ }^{6}$, Kenneth J. Takeuchi ${ }^{1,3}$, Esther S. Takeuchi ${ }^{1,3,5}$, Yimei Zhu ${ }^{2}$ \& Eric A. Stach (1) ${ }^{7}$

Spinel transition metal oxides (TMOs) have emerged as promising anode materials for lithium-ion batteries. It has been shown that reducing their particle size to nanoscale dimensions benefits overall electrochemical performance. Here, we use in situ transmission electron microscopy to probe the lithiation behavior of spinel $\mathrm{ZnFe}_{2} \mathrm{O}_{4}$ as a function of particle size. We have found that $\mathrm{ZnFe}_{2} \mathrm{O}_{4}$ undergoes an intercalation-to-conversion reaction sequence, with the initial intercalation process being size dependent. Larger $\mathrm{ZnFe}_{2} \mathrm{O}_{4}$ particles $(40 \mathrm{~nm})$ follow a two-phase intercalation reaction. In contrast, a solid-solution transformation dominates the early stages of discharge when the particle size is about 6-9 $\mathrm{nm}$. Using a thermodynamic analysis, we find that the size-dependent kinetics originate from the interfacial energy between the two phases. Furthermore, the conversion reaction in both large and small particles favors $\{111\}$ planes and follows a core-shell reaction mode. These results elucidate the intrinsic mechanism that permits fast reaction kinetics in smaller nanoparticles.

\footnotetext{
${ }^{1}$ Department of Materials Science and Chemical Engineering, SUNY-Stony Brook University, Stony Brook, NY 11794, USA. ${ }^{2}$ Department of Condensed Matter Physics, Brookhaven National Laboratory, Upton, NY 11973, USA. ${ }^{3}$ Department of Chemistry, SUNY-Stony Brook University, Stony Brook, NY 11794 , USA. ${ }^{4}$ Materials Science and Engineering Program and Department of Mechanical Engineering, The University of Texas at Austin, Austin, TX 78712, USA. ${ }^{5}$ Energy Sciences Directorate, Brookhaven National Laboratory, Interdisciplinary Sciences Building, Building 734, Upton, NY 11973, USA. ${ }^{6}$ Center for Functional Nanomaterials, Brookhaven National Laboratory, Upton, NY 11973, USA. ${ }^{7}$ Department of Materials Science and Engineering, University of Pennsylvania, Philadelphia, PA 19104, USA. Correspondence and requests for materials should be addressed to E.A.S. (email: stach@seas.upenn.edu)
} 
T here is an ever-increasing demand for lithium-ion batteries that have both large energy density and improved cycling life. With respect to anode materials, the most competitive candidates are those that exhibit alloying and/or conversion reactions because they can provide a much larger capacity than carbonaceous anode materials. A primary challenge to the adoption of these electrode materials is the huge capacity loss that they experience during cycling, which is generally attributed to the strain induced by the large volume changes that occur during lithiation and delithiation. Prior studies have shown that reducing particle size can help to relax the strain, and that this can lead to improved cyclability ${ }^{1,2}$. Reducing materials to nanoscale dimensions also leads to a high surface-to-volume ratio and reduced transport length ${ }^{3,4}$. Thus, there have been substantial efforts devoted to the development of nano-sized electrode materials ${ }^{5-14}$. Recent studies regarding lithiation of intercalation compounds have indicated that the intrinsic ionic/electronic transport behaviors can be tuned by controlling particle size ${ }^{15-18}$. Kobayashi et al. found that the miscibility gap of $\mathrm{LiFePO}_{4}$ cathode materials shrinks as particle size reduces, which results in a homogeneous single-phase transformation pathway for small particles $(<100 \mathrm{~nm})^{19}$. To date, a single-phase transformation pathway in nano-sized electrodes has only been observed in $\mathrm{LiFePO}_{4}$ and the relationship between particle size and reaction pathway has not been clarified in conversion-type electrode materials.

Spinel transition metal oxides (TMOs) have been shown to be a high specific capacity anode material for lithium-ion batteries ${ }^{20-22}$. Among TMOs, $\mathrm{Fe}_{3} \mathrm{O}_{4}$ has received significant attention due to its nontoxicity, low cost, and high electronic conductivity ${ }^{23-25}$. However, reduced $\mathrm{Fe}^{0}$ is not electrochemically active with $\mathrm{Li}^{+}$. Compounds in which another electroactive transition metal ion is substituted for one iron atom in the $\mathrm{Fe}_{3} \mathrm{O}_{4}$ structure have recently gained attention ${ }^{14,26-28}$. Zinc ferrite $\left(\mathrm{ZnFe}_{2} \mathrm{O}_{4}\right)$, for example, has a theoretical capacity of $1000 \mathrm{mAhg}^{-1}$, an improvement over $\mathrm{Fe}_{3} \mathrm{O}_{4}$ that results from the fact that metallic $\mathrm{Zn}$ can further alloy with additional $\mathrm{Li}^{+}$after the conversion reaction has occurred ${ }^{29}$. The process of lithium insertion into spinel zinc ferrite $\left(\left[\mathrm{Zn}^{2+}\right]_{8 \mathrm{a}}\left[\mathrm{Fe}^{3+}{ }_{2}\right]_{16 \mathrm{~d}} \mathrm{O}_{4}\right.$, denoted by the Wyckoff notation) has been studied by Waszczak et al. using X-ray diffraction ${ }^{30}$, and can be expressed as: $\left[\mathrm{Zn}^{2+}\right]_{8 \mathrm{a}}\left[\mathrm{Fe}^{3+}{ }_{2}\right]_{16 \mathrm{~d}} \mathrm{O}_{4}+\mathrm{Li}^{+} \rightarrow\left[\mathrm{Li}^{+} \mathrm{Zn}^{2+}\right]_{16 \mathrm{c}}$ $\left[\mathrm{Fe}^{3+}{ }_{2}\right]_{16 \mathrm{~d}} \mathrm{O}_{4}+7 \mathrm{Li}^{+} \rightarrow \mathrm{Zn}^{0}+\mathrm{Fe}^{0}+4 \mathrm{Li}_{2} \mathrm{O}$. Previous studies have reported that $\mathrm{ZnFe}_{2} \mathrm{O}_{4}$ powder and thin films suffer poor capacity reversibility and limited capacity when they have a particle size that is of the micron scale ${ }^{31,32}$. A more recent study found that even when the particles have nanoscale dimensions $(<15 \mathrm{~nm})$, the rate performance and cyclability of $\mathrm{ZnFe}_{2} \mathrm{O}_{4}$ still varied significantly with particle size ${ }^{33}$. These results indicate that in order to improve electrochemical performance it is crucial to explore the origin of particle size effects on reaction kinetics and elucidate the reaction mechanism.

To this end, we utilize the in situ dry cell transmission electron microscopy (TEM) technique, an approach which allows direct observation of structural changes at the nano- and atomic scale in real time $e^{34-38}$. Because of the absence of liquid electrolyte, the dry cell configuration enables high spatial resolution, which in turn allows real-time high-resolution TEM (HRTEM) imaging at atomic resolution. Moreover, because these HRTEM images can be subsequently converted to diffractograms using the Fast Fourier transforms (FFT) technique, in situ HRTEM imaging is capable of visualizing phase evolution and correlating it directly with morphological changes. In this work, we investigate the kinetics of small $(6-9 \mathrm{~nm})$ vs. large (ca. $40 \mathrm{~nm}) \mathrm{ZnFe}_{2} \mathrm{O}_{4}$ nanoparticles using in situ TEM. Although both the large and small $\mathrm{ZnFe}_{2} \mathrm{O}_{4}$ nanoparticles undergo both an intercalation and a subsequent conversion reaction process, we find that the lithiation pathways of the intercalation process varies with particle size: the small nanoparticles show a solid-solution behavior while the large nanoparticles undergo a two-phase intercalation reaction. We explain the origin of the size-dependent kinetics using a straightforward thermodynamic theory.

\section{Results}

Materials characterization and electrochemical properties. Zinc ferrite $\left(\mathrm{ZnFe}_{2} \mathrm{O}_{4}\right.$, abbreviated as $\mathrm{ZFO}$ hereafter $)$ nanoparticles with different crystalline sizes were synthesized (Fig. 1). The small ZFO (Fig. 1a) nanoparticles are $6-9 \mathrm{~nm}$ in size while the large ZFO (Fig. 1c) are of $\sim 40 \mathrm{~nm}$ in size, as illustrated in Fig. 1f. Both the small and large nanoparticles have the spinel structure, with the Fd $\overline{3} \mathrm{~m}$ Space Group, as confirmed by X-ray diffraction (Supplementary Figure 1) and corresponding selected area electron diffraction (SAED) patterns, shown respectively in Fig. 1b, d. In addition, the overall sample is polycrystalline, with individual particles having high crystallinity, as indicated by the atomicresolution high-angle annular dark field (HAADF) image shown in Fig. 1e. In order to understand the electrochemical performance of ZFO as a function of particle size, both large and small ZFO were discharged to $0.01 \mathrm{~V}$ at a rate of $200 \mathrm{mAg}^{-1}$. Theoretically, ZFO can take up to eight $\mathrm{Li}^{+}$per formula during the conversion reaction. As shown in Fig. 1g, both large and small ZFO receive more than eight $\mathrm{Li}^{+}$at the end of initial discharge: this excess is generally ascribed to the presence of side reactions, electrolyte decomposition and solid-electrolyte interface formation $^{39}$. When fully lithiated, the pristine ZFO, in both cases, has evolved into a nanocomposite composed of ultrafine $\mathrm{Fe}^{0}$ and $\mathrm{Zn}^{0}$ nanoparticles embedded in a matrix of amorphous $\mathrm{Li}_{2} \mathrm{O}$ : this has been confirmed by ex situ TEM (Supplementary Figures 2 and 3). The overall lithiation reaction is independent of particle size and can be expressed as: $\mathrm{ZnFe}_{2} \mathrm{O}_{4}+8 \mathrm{Li}^{+}+8 \mathrm{e}^{-} \rightarrow \mathrm{Zn}^{0}+2 \mathrm{Fe}^{0}+$ $4 \mathrm{Li}_{2} \mathrm{O}$. As shown in Fig. 1g, distinct plateaus appear as the lithiation reaction proceeds. Previous studies have assigned each plateau to specific lithiation processes $29,40,41$ : (1) $\mathrm{Li}^{+}$initially inserted into $16 \mathrm{c}$ sites $(\sim 1.64 \mathrm{~V})$, (2) further incoming $\mathrm{Li}^{+}$ forcing the $\mathrm{Zn}^{2+}$ at the $8 \mathrm{a}$ site to neighboring empty $16 \mathrm{c}$ sites $(\sim 1.58 \mathrm{~V})$, and $(3)$ the generation of the final discharge product $(<1 \mathrm{~V})$. The major difference in the kinetic response of the small ZFO (S-ZFO) and large ZFO (L-ZFO) occurs at the lower depth of discharge (DOD), which is associated with the $\mathrm{Li}^{+}$intercalation process. To investigate the mass transfer in detail, we utilized a galvanostatic intermittent titration technique (GITT) test at a rate of $62.5 \mathrm{mAg}^{-1}$ to measure the open-circuit voltage (OCV) at different DOD, as depicted in the inset of Fig. $1 \mathrm{~g}$ and Supplementary Figure 4. The OCV reflects the redox potential at the equilibrium state, whereby L-ZFO undergoes a two-step reaction with the formation of an intermediate phase. In contrast, the smooth decrease of the OCV in S-ZFO suggests the existence of a continuous, homogeneous single phase. However, every electrochemical reaction happens at a certain overpotential: thus, thermodynamics is not the only factor that controls the reaction pathway. In order to achieve an accurate understanding of the lithiation pathways, we have utilized in situ electron microscopy techniques to probe the reaction kinetics during operation ${ }^{42,43}$.

Phase evolution investigated by in situ electron diffraction. We utilize in situ electron diffraction to probe the phase evolution of both samples throughout the entire lithiation process. In Fig. 2, we plot the radially integrated intensity profiles of S-ZFO and LZFO as a function of reaction time, respectively, as extracted from their corresponding time-sequenced SAED patterns (see also Supplementary Figure 5 and Supplementary Movie 1 and 2). In the pristine state, the 111 peak of S-ZFO is missing after background subtraction (as shown in the bottom of Fig. 2a). This is 

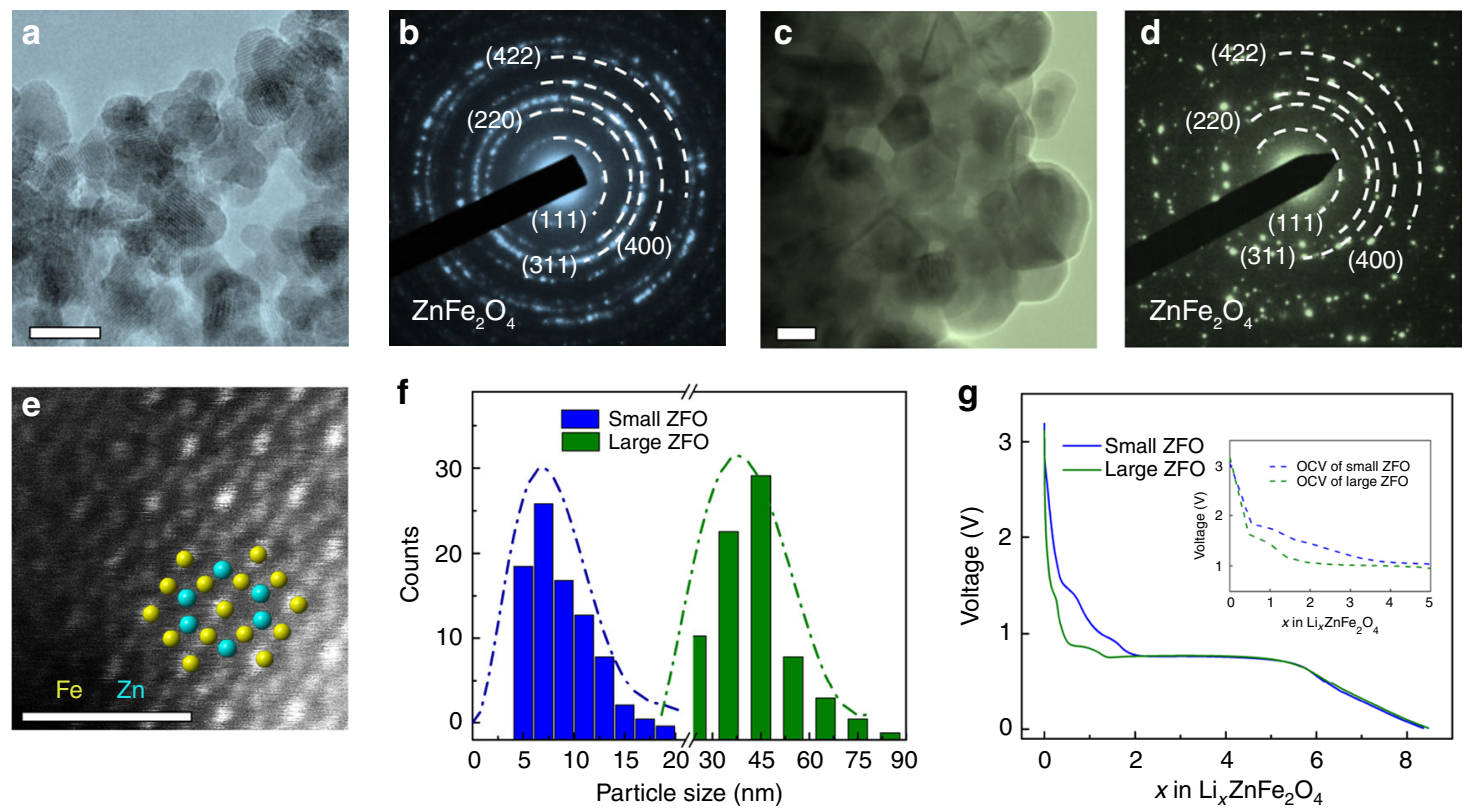

Fig. 1 Characterization of pristine materials and electrochemical properties. a Typical morphology of S-ZFO (scale bar: $10 \mathrm{~nm}$ ) and $\mathbf{b}$ the corresponding SAED pattern. c Typical morphology of L-ZFO (scale bar: $10 \mathrm{~nm}$ ) and $\mathbf{d}$ the corresponding SAED pattern. e HAADF-STEM image showing the spinel structure along the <101> zone axis, compared to an overlaid atomic model along the same projection. Scale bar: $1 \mathrm{~nm}$. f Size distribution of S-ZFO (blue) and L-ZFO (green). $\mathbf{g}$ Discharge profile of S-ZFO (blue) and L-ZFO (green) measured at the rate of $200 \mathrm{mAg}^{-1}$. Inset shows the OCV profile of S-ZFO and L-ZFO, respectively
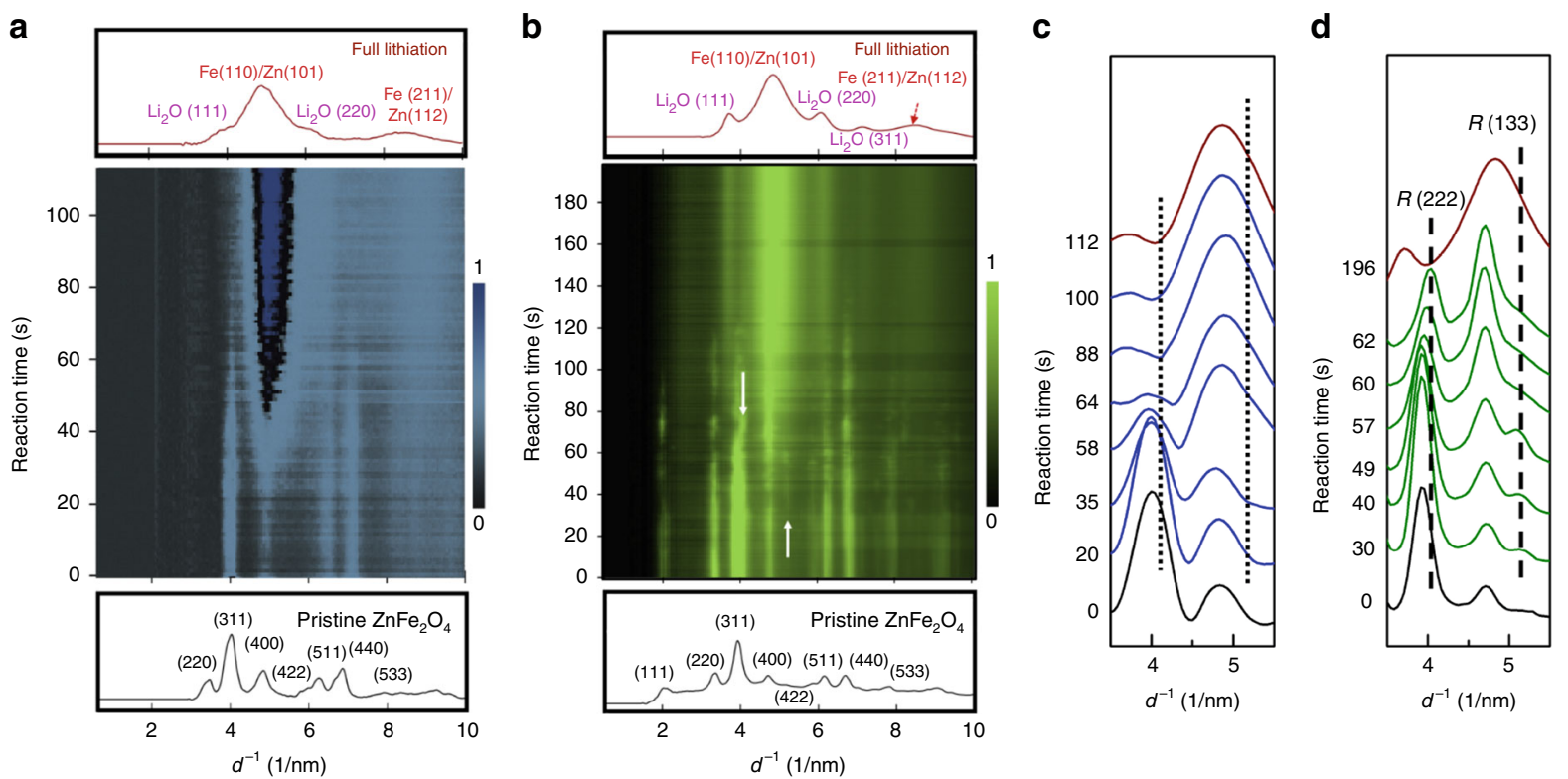

Fig. 2 Phase evolution tracked by in situ electron diffraction. Electron diffraction intensity profiles of a S-ZFO and $\mathbf{b}$ L-ZFO as a function of reaction time. The corresponding radial intensity spectrum of pristine (black) and fully lithiated (red) states are shown below and above the color map, respectively. c Radially integrated intensity profiles of S-ZFO as function of reaction time. The dotted lines point out the corresponding $d$-spacing of ordered rock-salt phase observed in $\mathbf{d}$. $\mathbf{d}$ Radially integrated intensity profiles of L-ZFO as a function of time. The additional phase indicated by white arrows in $\mathbf{b}$ corresponds to the ordered rock-salt phase $(R(222)$ and $R(133))$, as indicated by black dashed lines

because the 111 peak is too dim and close to the transmitted spot, which overwhelms the signal from it. For better visualization, we false-color the intensity profile (see Supplementary Figure 5) of SZFO with blue (Fig. 2a) and L-ZFO with green (Fig. 2b). For both samples, the initial sharp Bragg reflections associated with spinel ZFO (bottom spectrum) gradually change with lithiation into broadened peaks associated with $\mathrm{Li}_{2} \mathrm{O}$ and $\mathrm{Zn}^{0} / \mathrm{Fe}^{0}$ (top spectrum). This is the same as is observed in the ex situ data, which validates the approach. It is worth noting that two additional peaks appear at the lower DOD for the L-ZFO $(<60 \mathrm{~s})$, indicated by the white arrows in Fig. 2b. For clearer visualization, we extract and display the time-sequential intensity profiles of L-ZFO in the range where the new phase occurs, as shown in Fig. 2d. Comparing with the pristine state (black curve) and the fully lithiated state (red curve), it can be seen that two distinct peaks emerge at $4.04 \mathrm{~nm}^{-1}$ and $5.08 \mathrm{~nm}^{-1}$, which are associated with 222 and 133 reflections of 
the ordered rock-salt phase $\left(\left[\mathrm{Li}_{x}+\mathrm{Zn}^{2+}\right]_{16 c}\left[\mathrm{Fe}^{3+2]}\right]_{16 \mathrm{~d}} \mathrm{O}_{4}\right.$, denoted by the Wyckoff notation). These are indicated by the black dashed line in Fig. 2d. The formation of the ordered rock-salt phase can be ascribed to the process of incoming $\mathrm{Li}^{+}$repelling $\left[\mathrm{Zn}^{2+}\right]_{8 \mathrm{a}}$ into neighboring empty $16 \mathrm{c}$ sites. The structure of the ordered rocksalt phase is equivalent to a standard rock-salt structure, where both kinds of cations equally occupy all of the $16 \mathrm{c}$ sites. Upon further lithiation, both peaks gradually decrease in intensity with the emergence of the $\mathrm{Li}_{2} \mathrm{O} 111$ and $\mathrm{Fe} 110$ / Zn 101 reflections, thereby indicating that material has undergone the conversion process. In summary, L-ZFO undergoes a two-step reaction during lithiation (intercalation, then conversion) whereby the ordered rock-salt intermediate phase is first formed, then subsequently decomposed. Intriguingly, in the S-ZFO, the additional reflections found at $4.04 \mathrm{~nm}^{-1}$ and $5.08 \mathrm{~nm}^{-1}$ in the L-ZFO are not present, as shown by the dotted lines in Fig. 2c. As the lithiation reaction proceeds in S-ZFO, the intensity of the Bragg reflections associating with the spinel phase monotonically decreases with increasing intensity of the reflections associated with $\mathrm{Fe} / \mathrm{Zn}$ and $\mathrm{Li}_{2} \mathrm{O}$ phases, indicating that the S-ZFO exhibits a "direct" conversion upon lithiation (Supplementary Figure 6).

Two-step reaction pathway in L-ZFO visualized by in situ HRTEM. In order to obtain a more comprehensive understanding of the lithiation process, the microstructural evolution of L-ZFO during lithiation is investigated in real space (Supplementary Movie 3). One general deficiency of in situ techniques is the inability to obtain information in both real space and reciprocal space at the same time ${ }^{44-48}$. However, local microstructural changes and phase evolution can be achieved quasisimultaneously via the use of in situ HRTEM, followed by subsequent use of FFT. Using this approach, detailed information is accessible at different DOD (see detailed phase analysis in Supplementary Information). For example, the corresponding FFT pattern (Fig. 3b) is produced along with the partially lithiated particle (Fig. 3a). The FFT pattern includes two sets of diffraction spots, which are associated with the spinel structure and ordered rock-salt structure, respectively (Fig. 3c). Using these two set of spots in the FFT, we can map the distribution of the spinel (green) and ordered rock-salt (magenta) phase within a single nanoparticle (Fig. 3d) by inverting the FFT with only this information preserved ${ }^{49}$. Similarly, by combining the timesequenced phase distribution maps with the corresponding HRTEM images, as shown in Fig. 3e (Supplementary Figure 7), we can track the evolution of both the phase information and the morphological information at the same time, within individual nanoparticles. We see here that the lithiation process is driven by $\mathrm{Li}^{+}$diffusion from the bottom of the image to the top. At the initial stage of discharge $(<492 \mathrm{~s})$, incoming $\mathrm{Li}^{+}$ions push $\left[\mathrm{Zn}^{2+}\right]_{8 \mathrm{a}}$ to nearby empty $16 \mathrm{c}$ sites, leading to a phase transformation from the spinel (green false color) to the ordered rock-salt phase (magenta false color). Further lithiation triggers the subsequent conversion reaction, during which the intermediate phase evolves into the final discharge products of ultra-small metallic $\mathrm{Zn}$ and $\mathrm{Fe}$ nanoparticles and lithium oxide. In addition, the conversion reaction is observed to generally propagate from the exterior surface (along $\{111\}$ planes) toward the particle center, whereas no preferential lithiation pathway is observed during the initial intercalation process. Based on observations in both real space and reciprocal space, we can conclude that the L-ZFO undergoes a two-step reaction, which initiates with random $\mathrm{Li}^{+}$ intercalation, followed by a "core-shell" pathway upon the subsequent conversion reaction.

Solid-solution transformation in S-ZFO visualized by in situ HRTEM. Similarly, we investigated the lithiation of S-ZFO in real time (Supplementary Movie 4). Unlike the two-phase reaction process observed in L-ZFO, S-ZFO exhibits a solid-solution transformation during lithiation, as shown in Fig. 4a. The S-ZFO nanoparticle retains the spinel structure (blue) until the completion of the conversion reaction, which is in agreement with previous in situ electron diffraction results (see Fig. 2a, c and detailed analysis in Supplementary Figure 6). In addition, we found the orientation of the particle changes during our in situ TEM observation. As shown in Fig. 4b (Supplementary Figure 8), the orientation of that particle changed to the $\langle 110\rangle$ zone axis at
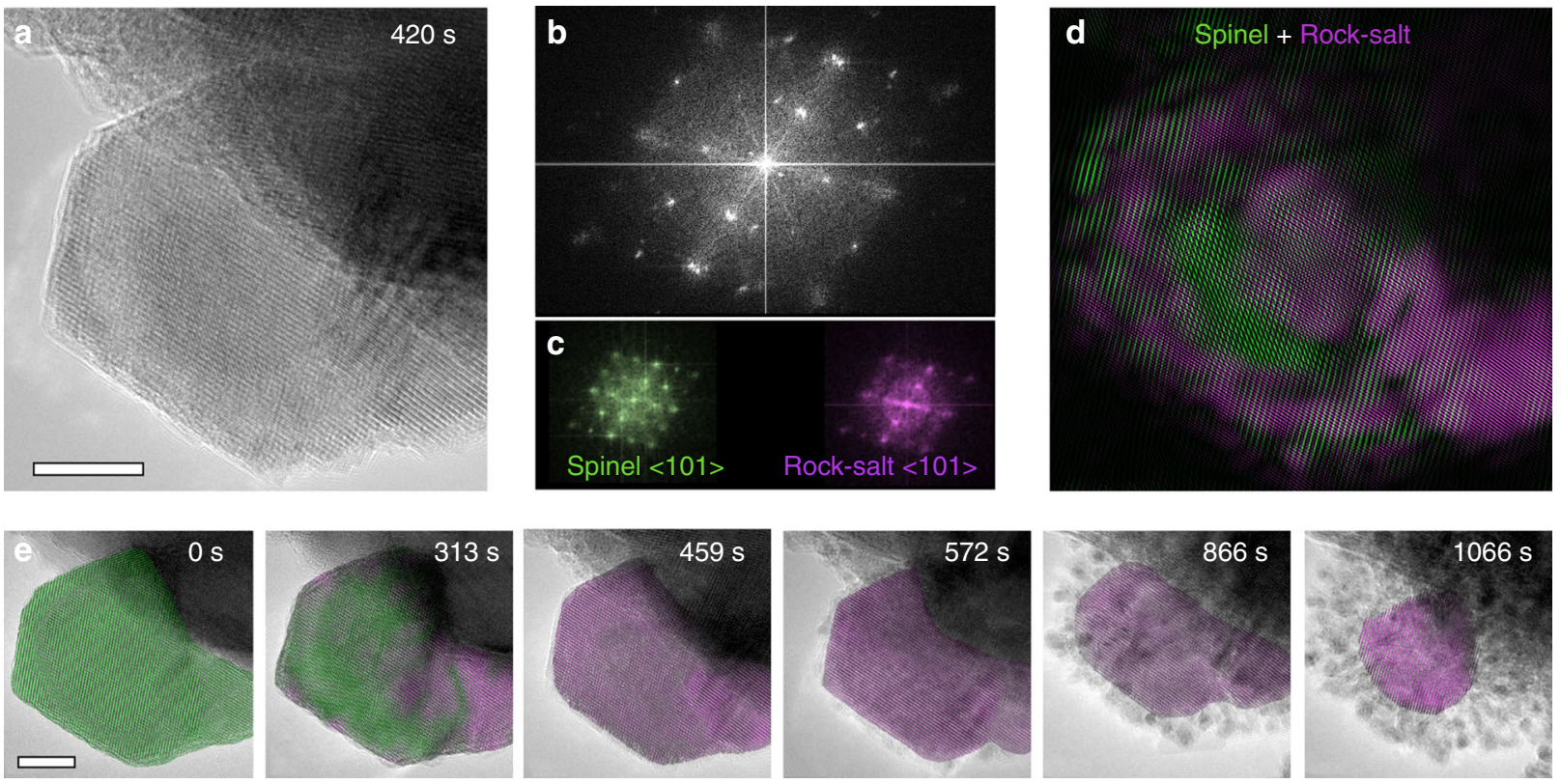

Fig. 3 Two-phase transformation mechanism probed via in situ HRTEM imaging. a HRTEM image of partially reacted L-ZFO obtained at $420 \mathrm{~s}$ (scale bar: $10 \mathrm{~nm}$ ) and $\mathbf{b}$ the corresponding FFT pattern. $\mathbf{c}$ Two sets of FFT patterns extracted from $\mathbf{b}$ representing coexistence of spinel (green) and ordered rock-salt phase (magenta). d Inverse FFT image shows the distribution of spinel (green) and ordered rock-salt phase (magenta) in real space. e Time-sequenced HRTEM images with phase information (false-color) showing the transformation pathway of L-ZFO as a function of reaction time. Scale bar: $10 \mathrm{~nm}$ 


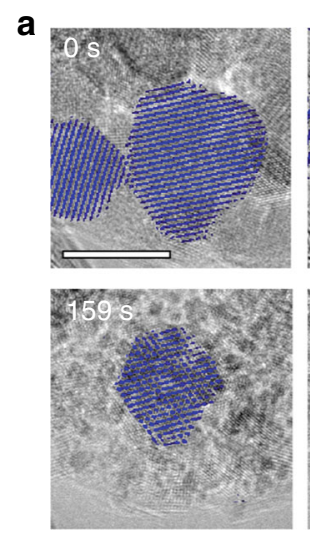

b

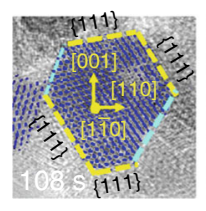

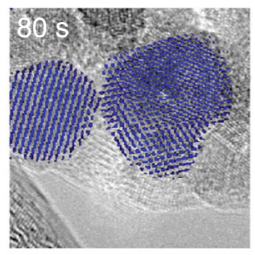

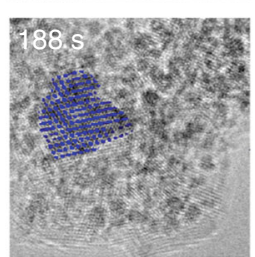

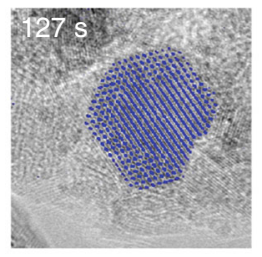
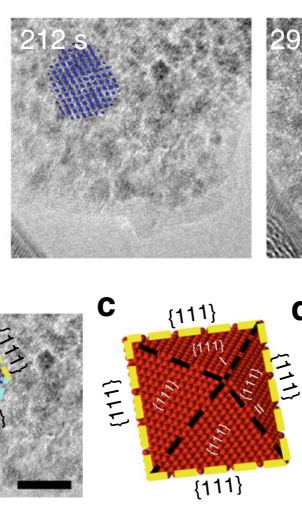
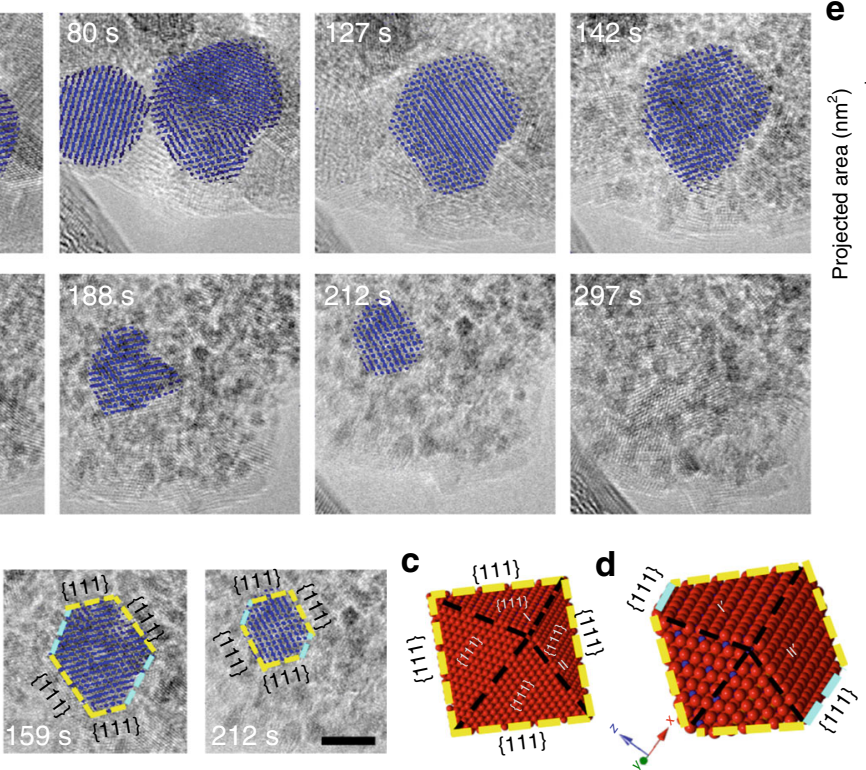
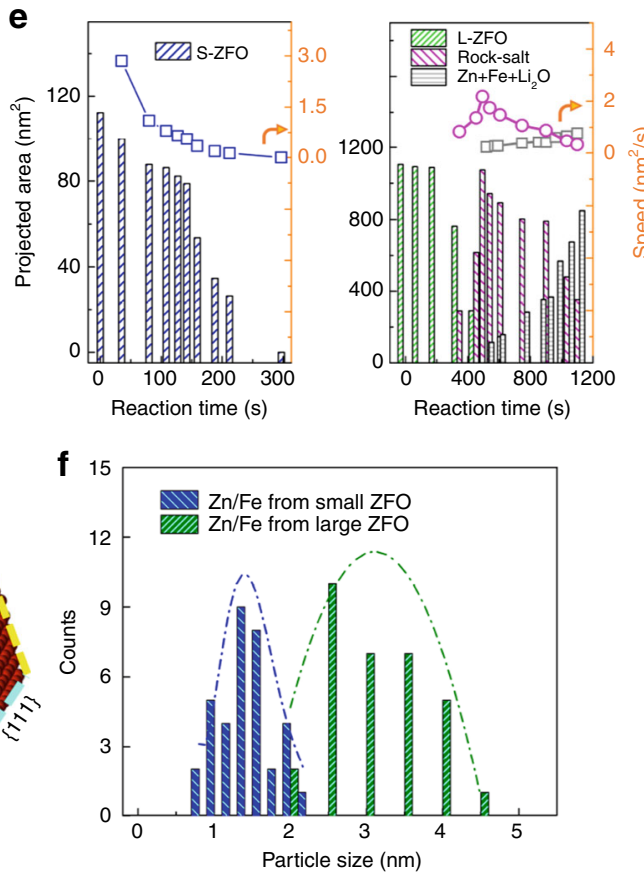

Fig. 4 In situ HRTEM study of S-ZFO and comparison of reaction kinetics of ZFO with different particle size. a Time-sequenced HRTEM images with phase information (overlaid false color) showing the phase evolution of S-ZFO as a function of reaction time. Scale bar: $10 \mathrm{~nm}$. $\mathbf{b}$ Enlarged HRTEM images illustrating the preferred reaction interface. $\mathbf{c}$ Atomic model of $\{111\}$ octahedron viewing along <110>direction. Scale bar: $5 \mathrm{~nm}$. $\mathbf{d}$ Atomic model showing the structure of partially reacted $\{111\}$ octahedron. e Projected area of spinel phase in S-ZFO (left panel, blue) and the three phases occurring in L-ZFO (right panel, green: L-ZFO, magenta: ordered rock-salt and gray: nanocomposite) changes as a function of time. Curves on the top of each panel showing the propagation speed of each reaction, which is the derivative of the projected area of each phase to reaction time (vertical axis on the right). $\mathbf{f}$ Size distribution of final discharge products $\left(\mathrm{Zn}^{0} / \mathrm{Fe}^{0}\right)$ generated from S-ZFO (blue) and L-ZFO (green), respectively

$108 \mathrm{~s}$ from the $<111>$ zone axis. It is interesting to note that by $108 \mathrm{~s}$ the exposed facets are $\{111\}$ planes and these facets were maintained until the particle was fully lithiated $(297 \mathrm{~s})$, as indicated by the yellow and turquoise dashed lines in Fig. 4b. The presence of preferential exposed $\{111\}$ facets is also observed in other S-ZFO particles (Supplementary Figure 9). An atomic model in Fig. 4c represents the projection of a ZFO with $\{111\}$ planes as the exposed facets, viewed along a $<110>$ direction. The model structures are rotated to match the experimental data and slightly tilted to permit visualization of the three-dimensional out-of-plane features. In projection, the partially reacted particle (Fig. $4 \mathrm{~b}$ ) is a truncated $\{111\}$ octahedron with two vertexes decomposed into a nanocomposite. Based on previous studies, the reconstructed $\{111\}$ surface planes are more stable than the $\{100\}$ surface planes, and it is preferable to have exposed facets that have more transition metal cations ${ }^{50-52}$. With respect to ZFO, the $\{111\}$ planes of ZFO have one more $\mathrm{Fe}^{3+}$ than the $\{100\}$ planes, suggesting a faster $\mathrm{Fe}^{3+} / \mathrm{Fe}^{0}$ redox reaction on the $\{111\}$ plane. This would lead to the formation of the "cropped" vertices (shown as the turquoise dashed line), which is where the intersection $\{111\}$ planes occurs. This broadly verifies that the $\{111\}$ planes are the most preferred reaction interface, as depicted in Fig. 4d.

With the assumption that the electrochemical condition within one single particle is identical, we measured the reaction kinetics of S-ZFO and L-ZFO, respectively, by quantifying the projected areas of each phase within individual particles as a function of reaction time (Fig. 4e). The propagation speed of the solidsolution reaction is found to be no greater than $3 \mathrm{~nm}^{2} / \mathrm{s}$. For the L-ZFO, the projected areas of three phases-spinel, ordered rocksalt, and nanocomposite-are measured, respectively. The propagation speed of the two-phase intercalation reaction is found to be one order of magnitude faster than the subsequent conversion reaction. In addition, S-ZFO generates finer metallic nanoparticles than those found in the nanocomposite created from L-ZFO (Fig. 4f). The presence of ultrafine-sized metallic Fe and $\mathrm{Zn}$ would further reduce the $\mathrm{Li}^{+}$diffusion length in subsequent cycles, which may lead to enhanced rate capability for electrode materials with small particle size.

\section{Discussion}

The aforementioned lithiation pathways of S-ZFO and L-ZFO are schematically illustrated in Fig. $5 a$, b, respectively. We found that L-ZFO undergoes a two-step reaction, in a manner similar to many other spinel TMOs ${ }^{24,30,53}$. The reaction mechanism can be expressed as:

$$
\begin{array}{r}
{\left[\mathrm{Zn}^{2+}\right]_{8 \mathrm{a}}\left[\mathrm{Fe}_{2}^{3+}\right]_{16 \mathrm{~d}} \mathrm{O}_{4} \rightarrow\left[\mathrm{Li}_{x}^{+} \mathrm{Zn}^{2+}\right]_{16 \mathrm{c}}\left[\mathrm{Fe}_{2}^{3+}\right]_{16 \mathrm{~d}} \mathrm{O}_{4} \rightarrow \mathrm{Zn}^{0}} \\
+\mathrm{Fe}^{0}+\mathrm{Li}_{2} \mathrm{O} .
\end{array}
$$

In addition, we conducted ex situ HRTEM imaging on ZFO at an early DOD $(x<1)$. A similar mixture of spinel and rock-salt phases was found in ex situ sample (Supplementary Fig. 10): this further demonstrates consistency between the in situ and ex situ results. Intriguingly, the S-ZFO does not follow the two-phase intercalation reaction pathway. The primary features of the twophase intercalation reaction-the formation of the ordered rocksalt intermediate phase and the corresponding volume change $(\sim 4.4 \%)-$ are not observed in S-ZFO upon lithiation. The negligible volume expansion we see in S-ZFO was also corroborated at the macroscopic scale via the in situ XRD technique ${ }^{41}$. Our results strongly suggest that S-ZFO follows a solid-solution transformation pathway, leading to a solid-solution phase $\left(\mathrm{Li}_{y} \mathrm{ZnFe}_{2} \mathrm{O}_{4}\right)$ as the initial state of lithiation, as depicted in Fig. $5 b$. 
a

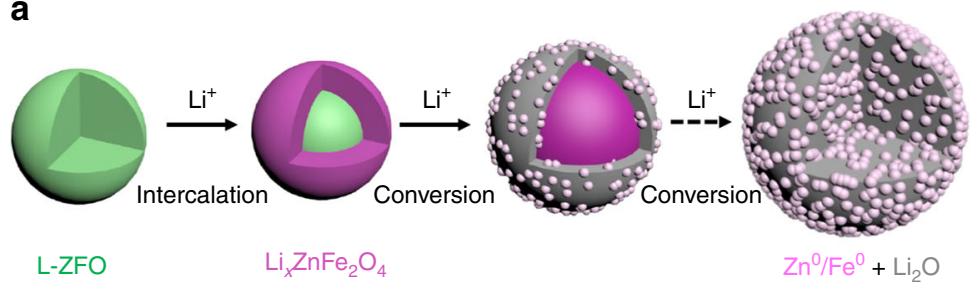

b

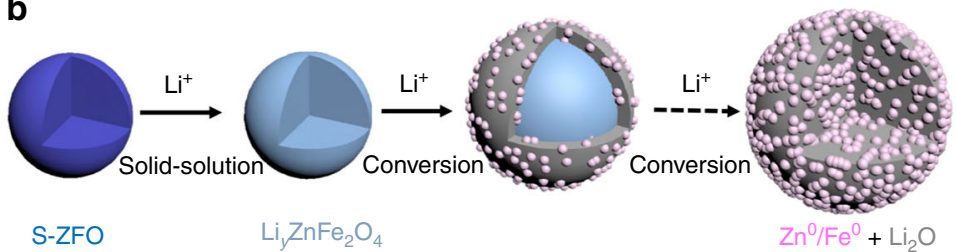

C

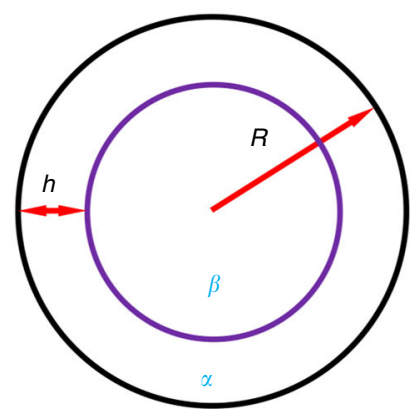

Fig. 5 Phase transformation pathway of L-ZFO and S-ZFO nanoparticles. Schematic 3D models illustrating the lithiation pathway of a L-ZFO and $\mathbf{b}$ S-ZFO. c Schematic diagram shows the coexistence of pre-existing phase $(\beta)$ and newly formed phase $(\alpha)$ inside a particle undergoing a core-shell reaction mode, where $R$ is the size of the particle and $h$ is the thickness of $\alpha$ phase coated on the $\beta$ phase

The differences between the intercalation pathways of the large and small ZFO may originate from the difference in interfacial energy between the pre-existing phase and the new phase. These concepts can be explored within a well-established thermodynamic theory: the phase separation from a homogenous concentration $x_{\varepsilon}$ to a mixture of $\alpha$ phase with concentration $x_{\alpha}$ and $\beta$ phase with concentration $x_{\beta}$ can happen only when the change of Gibbs free energy is negative ${ }^{54}$. The change of Gibbs free energy $(\Delta G)$ can be expressed as Eq. (2):

$$
\Delta G=V_{a} g_{a}\left(x_{a}\right)+V_{\beta} g_{\beta}\left(x_{\beta}\right)+S_{\alpha} \sigma_{\alpha}+S_{\beta} \sigma_{\beta}+S_{\alpha \beta} \gamma-V g_{\varepsilon}\left(x_{\varepsilon}\right) .
$$

where the $V$ is the volume of the crystallite, $g_{n}\left(x_{n}\right)$ is the free energy of the phase per unit volume, $x_{n}$ is the concentration of solute atoms of each phase, $S$ is the surface area, $\sigma$ is surface energy and $\gamma$ is the energy of the interface between the $\alpha$ and $\beta$ phases, assuming the surface energy and the volume of a particle remain the same during the phase transformation $(\varepsilon \rightarrow \alpha+\beta)$. Conventionally, the surface and interfacial energy terms in Eq. (2) do not contribute significantly since the particle size is large. However, as the particle size decreases, the contribution of the interfacial energy is no longer negligible. This is because the driving force for the transformation scales with volume, while the interfacial energy-which hinders the transformation-scales with area. Based on our in situ observations, we can see that the phase transformation of ZFO proceeds via a core-shell mode (Fig. 5c). Thus, the associated change of Gibbs free energy is as follows:

$$
\begin{array}{r}
\Delta G=\frac{4 \pi}{3}\left[R^{3}-(R-h)^{3}\right] g_{\alpha}\left(x_{\alpha}\right)+\frac{4 \pi}{3}(R-h)^{3} g_{\beta}\left(x_{\beta}\right) \\
+4 \pi(R-h)^{2} \gamma-\frac{4 \pi}{3} R^{3} g_{\varepsilon}\left(x_{\varepsilon}\right)
\end{array}
$$

in which

$$
h=R-R\left(\frac{x_{\alpha}-x_{\varepsilon}}{x_{\alpha}-x_{\beta}}\right)^{\frac{1}{3}},
$$

as determined by the lever rule. From Eqs. (3) and (4), we find the critical particle size to be:

$$
R \geq \frac{3 \gamma\left(\frac{x_{\alpha}-x_{\varepsilon}}{x_{\alpha}-x_{\beta}}\right)^{\frac{2}{3}}}{\Delta g_{\mathrm{v}}}
$$

that leads to the phase separation $(\Delta G \leq 0)$, where $\Delta g_{\mathrm{v}}$ is the change of free energy per unit volume after the phase transformation. In other words, particles less than the critical size $\left(R^{*}=\frac{3 y\left(\frac{x_{\alpha}-x_{\varepsilon}}{x_{\alpha}-x_{\beta}}\right)^{\frac{2}{3}}}{\Delta g_{\mathrm{v}}}\right)$ will undergo a solid-solution transformation, without the formation of the ordered rock-salt $\mathrm{Li}_{x} \mathrm{ZnFe}_{2} \mathrm{O}_{4}$. This is consistent with our in situ TEM observations of the S-ZFO particles, where we only observed a direct conversion process. For the solid-solution process, the reaction kinetics are not limited by the migration speed of phase boundary: this fact may benefit the cycling performance. It is also worth noting that analysis above regarding the size-dependent interfacial energy can be extended to other intercalation oxide electrode materials. Furthermore, during the conversion process, we observed a core-shell reaction mode. The volume expansion induced by the conversion reaction (the shell) produces a compressive strain on the core which can retard further reaction ${ }^{55}$. In other words, the smaller particle size ZFO particles are, the less compressive strain they suffer. Therefore, during both intercalation and conversion processes, the reaction kinetics are enhanced by small particle sizes.

In summary, we have investigated the lithiation pathway of spinel zinc ferrite as a function of particle size using in situ TEM techniques. We have found that below a critical size $\left(9 \mathrm{~nm}<R^{\star}\right.$ $<40 \mathrm{~nm})$ the two-phase reaction pathway is suppressed and a solid-solution process dominates the intercalation reaction, which is analogous to the case of $\mathrm{LiFePO}_{4}$. Moreover, we have found that the enhanced electrochemical performance of small electrode materials is not only a result of the reduced $\mathrm{Li}^{+}$and $\mathrm{e}^{-}$diffusion length but also benefits from the modification of both the electrochemical reaction pathway and the reduction of the strain during the reaction. In addition, we found the subsequent conversion reaction prefers to proceed via $\{111\}$ planes regardless of particle size: this indicates that crystallographic structure also strongly impacts electrochemical performance and $\mathrm{Li}^{+}$transport. These results suggest a rational approach for improving the electrochemical performance of other conversion-type electrode materials and provide a criterion for the design and selection of 
spinel TMOs as anode materials with enhanced rate capability and cycling life.

\section{Methods}

Sample preparation. $\mathrm{ZnFe}_{2} \mathrm{O}_{4}$ nanomaterials were prepared using a coprecipitation method. Briefly, stoichiometric solutions of $\mathrm{Zn}$ - and Fe-based nitrate salts ( $\mathrm{Zn}$ $\left.\left(\mathrm{NO}_{3}\right)_{2}, \mathrm{Fe}\left(\mathrm{NO}_{3}\right)_{3}\right)$ were added concurrently to a deionized water solution containing excess triethylamine in an ice bath. The precipitate was collected and vacuum-dried. For small $\mathrm{ZnFe}_{2} \mathrm{O}_{4}$, the precipitate was treated hydrothermally at $120^{\circ} \mathrm{C}$ for $12 \mathrm{~h}$ using DI water as solvent. The small $\mathrm{ZnFe}_{2} \mathrm{O}_{4}$ was further prepared by using graphene oxide as a template and Pluronic copolymers with 4400 molecular weight as surfactants. The large $\mathrm{ZnFe}_{2} \mathrm{O}_{4}$ sample is obtained by heat treatment of the precipitate at $500^{\circ} \mathrm{C}$ for $6 \mathrm{~h}$. The final sample is washed and vacuum-dried.

Electrochemical measurements. The composite electrode used for electrochemical measurements were prepared with $80 \mathrm{wt} \%$ active material, $10 \mathrm{wt} \%$ carbon black, and $10 \mathrm{wt} \%$ polyvinylidene fluoride in $\mathrm{N}$-methyl-2-pyrrolidone and cast onto a copper foil current collector. R2032-type coin cells were assembled inside an argon-filled glove box with the as-prepared composite electrode as cathode and $\mathrm{Li}$ metal as anode. A Celgard 2400 monolayer polyethylene separator and $1 \mathrm{M}$ lithium hexafluorophosphate $\left(\mathrm{LiPF}_{6}\right)$ solution in ethylene carbonate:dimethyl carbonate (DMC) (1:1 in weight) were used as the electrolyte for coin cells. Battery testing was performed using a battery test station (Arbin BT2000) at room temperature. Each current pulse performed during the GITT measurements was followed by a $24-\mathrm{h}$ relaxation period to ensure full relaxation of the OCV.

TEM characterization. The in situ dry cell was assembled into a Nanofactory STM specimen holder inside an argon-filled glove box. The electrochemical cell is composed of three parts: (1) metallic Li that is coated on a piezo-driven tungsten tip that functions as an anode, (2) $\mathrm{Li}_{2} \mathrm{O}$ formed on the surface of $\mathrm{Li}$ anode which functions as a solid electrolyte and (3) $\mathrm{ZnFe}_{2} \mathrm{O}_{4}$ powder dispersed on a half TEM grid with amorphous carbon support work which functions as the positive electrode. After installation, the holder is transferred to the TEM column within a sealed, argon-filled bag in order to avoid air exposure. During operation, a constant negative DC potential was applied between the positive electrode and the Li source in a range of $3 \sim 5 \mathrm{~V}$ (discharge). The lithiation process was observed in real time by TEM imaging or diffraction mode. The ex situ samples after cycling were directly removed from the coin cell to a DMC solution inside an argon-filled glove box. For sample preparation, the cycled materials were sonicated and dispersed on a TEM grid. In situ and ex situ TEM characterization were done on JEOL JEM-2100F transmission electron microscope equipped with a field-emission electron gun that operated at $200 \mathrm{kV}$. Analytical EELS and high-resolution HAADF imaging were performed at a Hitachi-2700C scanning transmission electron microscope operated at $200 \mathrm{kV}$, which is equipped with cold field-emission gun and a probe aberration corrector, yielding a spatial and energy resolution down to $1 \AA$ and $0.35 \mathrm{eV}$, respectively.

\section{Data availability}

All data generated or analyzed during this study are included in this published Article and its Supplementary Information files. Further information is also available from the corresponding author upon reasonable request.

Received: 22 April 2018 Accepted: 9 November 2018

Published online: 09 January 2019

\section{References}

1. Liu, X. H. et al. Size-dependent fracture of silicon nanoparticles during lithiation. ACS Nano 6, 1522-1531 (2012).

2. Komaba, S. et al. Electrochemical Na insertion and solid electrolyte interphase for hard-carbon electrodes and application to Na-ion batteries. Adv. Funct. Mater. 21, 3859-3867 (2011).

3. Aricò, A. S., Bruce, P., Scrosati, B., Tarascon, J. M. \& Van Schalkwijk, W. Nanostructured materials for advanced energy conversion and storage devices. Nat. Mater. 4, 366-377 (2005).

4. Poizot, P., Laruelle, S., Grugeon, S., Dupont, L. \& Tarascon, J. M. Nano-sized transition-metal oxides as negative-electrode materials for lithium-ion batteries. Nature 407, 496-499 (2000).

5. Poizot, P., Laruelle, S., Grugeon, S., Dupont, L. \& Tarascon, J. M. From the vanadates to 3d-metal oxides negative electrodes. Ionics 6, 321-330 (2000).

6. Ji, L., Lin, Z., Alcoutlabi, M. \& Zhang, X. Recent developments in nanostructured anode materials for rechargeable lithium-ion batteries. Energy Environ. Sci. 4, 2682 (2011).
7. Luo, L. et al. Surface coating constraint induced self-discharging of silicon nanoparticles as anodes for lithium ion batteries. Nano Lett. 15, 7016-7022 (2015).

8. Shi, Y. et al. A tunable 3D nanostructured conductive gel framework electrode for high-performance lithium ion batteries. Adv. Mater. 29, 1603922 (2017).

9. Zhang, W. M., Wu, X. L., Hu, J. S., Guo, Y. G. \& Wan, L. J. Carbon coated $\mathrm{Fe}_{3} \mathrm{O}_{4}$ nanospindles as a superior anode material for lithium-ion batteries. Adv. Funct. Mater. 18, 3941-3946 (2008).

10. Liu, N. et al. A yolk-shell design for stabilized and scalable li-ion battery alloy anodes. Nano Lett. 12, 3315-3321 (2012).

11. Hwang, T. H., Lee, Y. M., Kong, B. S., Seo, J. S. \& Choi, J. W. Electrospun core-shell fibers for robust silicon nanoparticle-based lithium ion battery anodes. Nano Lett. 12, 802-807 (2012).

12. Taberna, P. L., Mitra, S., Poizot, P., Simon, P. \& Tarascon, J. M. High rate capabilities $\mathrm{Fe}_{3} \mathrm{O}_{4}$-based $\mathrm{Cu}$ nano-architectured electrodes for lithium-ion battery applications. Nat. Mater. 5, 567-573 (2006).

13. Park, S. J. et al. Side-chain conducting and phase-separated polymeric binders for high-performance silicon anodes in lithium-ion batteries. J. Am. Chem. Soc. 137, 2565-2571 (2015).

14. Xing, Z., Ju, Z., Yang, J., Xu, H. \& Qian, Y. One-step hydrothermal synthesis of $\mathrm{ZnFe}_{2} \mathrm{O}_{4}$ nano-octahedrons as a high capacity anode material for Li-ion batteries. Nano Res. 5, 477-485 (2012).

15. Bruce, P. G., Scrosati, B. \& Tarascon, J. M. Nanomaterials for rechargeable lithium batteries. Angew. Chem. Int. Ed. 47, 2930-2946 (2008).

16. Malik, R., Burch, D., Bazant, M. \& Ceder, G. Particle size dependence of the ionic diffusivity. Nano Lett. 10, 4123-4127 (2010).

17. Malik, R., Zhou, F. \& Ceder, G. Kinetics of non-equilibrium lithium incorporation in $\mathrm{LiFePO}_{4}$. Nat. Mater. 10, 587-590 (2011).

18. Zhu, C. et al. Size-dependent staging and phase transition in $\mathrm{LiFePO}_{4} / \mathrm{FePO}_{4}$. Adv. Funct. Mater. 24, 312-318 (2014).

19. Kobayashi, G. et al. Isolation of solid solution phases in size-controlled $\mathrm{Li} x \mathrm{FePO}_{4}$ at room temperature. Adv. Funct. Mater. 19, 395-403 (2009).

20. Ferg, E., Gummow, R. J., De Kock, A. \& Thackeray, M. M. Spinel anodes for lithium-ion batteries. J. Electrochem. Soc. 141, L147-L150 (1994).

21. Palacín, M. R. Recent advances in rechargeable battery materials: a chemist's perspective. Chem. Soc. Rev. 38, 2565-2575 (2009).

22. Tarascon, J. M. \& Armand, M. Issues and challenges facing rechargeable lithium batteries. Nature 414, 359-367 (2001).

23. Ban, C. et al. Nanostructured $\mathrm{Fe}_{3} \mathrm{O}_{4} / \mathrm{SWNT}$ electrode: binder-free and highrate li-ion anode. Adv. Mater. 22, E145-E149 (2010).

24. Thackeray, M. M., David, W. I. F. \& Goodenough, J. B. Structural characterization of the lithiated iron oxides $\mathrm{Li}_{x} \mathrm{Fe}_{3} \mathrm{O}_{4}$ and $\mathrm{Li}_{x} \mathrm{Fe}_{2} \mathrm{O}_{3}(0<\mathrm{x}<2)$. Mater. Res. Bull. 17, 785-793 (1982).

25. Thackeray, M. M., David, W. I. F. \& Goodenough, J. B. High-temperature lithiation of $\alpha-\mathrm{Fe}_{2} \mathrm{O}_{3}$ : a mechanistic study. J. Solid State Chem. 55, 280-286 (1984).

26. Bomio, M., Lavela, P. \& Tirado, J. L. Electrochemical evaluation of $\mathrm{CuFe}_{2} \mathrm{O}_{4}$ samples obtained by sol-gel methods used as anodes in lithium batteries. $J$. Solid State Electrochem. 12, 729-737 (2008).

27. Xing, Z., Ju, Z., Yang, J., Xu, H. \& Qian, Y. One-step solid state reaction to selectively fabricate cubic and tetragonal $\mathrm{CuFe}_{2} \mathrm{O}_{4}$ anode material for high power lithium ion batteries. Electrochim. Acta 102, 51-57 (2013).

28. Lavela, P. \& Tirado, J. L. $\mathrm{CoFe}_{2} \mathrm{O}_{4}$ and $\mathrm{NiFe}_{2} \mathrm{O}_{4}$ synthesized by sol-gel procedures for their use as anode materials for $\mathrm{Li}$ ion batteries. J. Power Sources 172, 379-387 (2007).

29. Bresser, D. et al. Carbon coated $\mathrm{ZnFe}_{2} \mathrm{O}_{4}$ nanoparticles for advanced lithiumion anodes. Adv. Energy Mater. 3, 513-523 (2013).

30. Chen, C., Greenblatt, M. \& Waszczak, J. Lithium insertion into spinel ferrites Solid State Ion. 18-19, 838-846 (1986).

31. NuLi, Y. N., Chu, Y. Q. \& Qin, Q. Z. Nanocrystalline $\mathrm{ZnFe}_{2} \mathrm{O}_{4}$ and Ag-doped $\mathrm{ZnFe}_{2} \mathrm{O}_{4}$ films used as new anode materials for Li-ion batteries. J. Electrochem. Soc. 151, A1077-A1083 (2004).

32. Sharma, Y., Sharma, N., Rao, G. V. S. \& Chowdari, B. V. R. Li-storage and cyclability of urea combustion derived $\mathrm{ZnFe}_{2} \mathrm{O}_{4}$ as anode for Li-ion batteries. Electrochim. Acta 53, 2380-2385 (2008).

33. Peng, L. et al. Two-dimensional holey nanoarchitectures created by confined self-assembly of nanoparticles via block copolymers: from synthesis to energy storage property. ACS Nano 12, 820-828 (2018).

34. Zhu, Y. et al. In situ atomic-scale imaging of phase boundary migration in $\mathrm{FePO}_{4}$ microparticles during electrochemical lithiation. Adv. Mater. 25, 5461-5466 (2013).

35. McDowell, M. T. et al. In situ TEM of two-phase lithiation of amorphous silicon nanospheres. Nano Lett. 13, 758-764 (2013).

36. He, K. et al. Transitions from near-surface to interior redox upon lithiation in conversion electrode materials. Nano Lett. 15, 1437-1444 (2015).

37. $\mathrm{Gu}, \mathrm{M}$. et al. In itu TEM study of lithiation behavior of silicon nanoparticles attached to and embedded in a carbon matrix. ACS Nano 6, 8439-8447 (2012). 
38. Li, J. et al. Kinetic phase evolution of spinel cobalt oxide during lithiation. ACS Nano 10, 9577-9585 (2016).

39. Hu, Y. Y. et al. Origin of additional capacities in metal oxide lithium-ion battery electrodes. Nat. Mater. 12, 1130-1136 (2013).

40. Guo, $\mathrm{H}$. et al. A first principles study of spinel $\mathrm{ZnFe}_{2} \mathrm{O}_{4}$ for electrode materials in lithium-ion batteries. Phys. Chem. Chem. Phys. 19, 26322-26329 (2017).

41. Zhang, Y. et al. Probing the Li insertion mechanism of $\mathrm{ZnFe}_{2} \mathrm{O}_{4}$ in Li-ion batteries: a combined X-ray diffraction, extended X-ray absorption fine structure, and density functional theory study. Chem. Mater. 29, 4282-4292 (2017).

42. Shang, T. et al. Atomic-scale monitoring of electrode materials in lithium-ion batteries using in situ transmission electron microscopy. Adv. Energy Mater. 7, 1700709 (2017).

43. Yuan, Y., Amine, K., Lu, J. \& Shahbazian-Yassar, R. Understanding materials challenges for rechargeable ion batteries with in situ transmission electron microscopy. Nat. Commun. 8, 15806 (2017).

44. Key, B. et al. Real-time NMR investigations of structural changes in silicon electrodes for lithium-ion batteries. J. Am. Chem. Soc. 131, 9239-9249 (2009).

45. Key, B., Morcrette, M., Tarascon, J. M. \& Grey, C. P. Pair distribution function analysis and solid state NMR studies of silicon electrodes for lithium ion batteries: understanding the (de)lithiation mechanisms. J. Am. Chem. Soc. 133, 503-512 (2011).

46. Bomio, M., Lavela, P. \& Tirado, J. L. ${ }^{57} \mathrm{Fe}$ Mössbauer spectroscopy and electron microscopy study of metal extraction from $\mathrm{CuFe}_{2} \mathrm{O}_{4}$ electrodes in lithium cells. Chemphyschem 8, 1999-2007 (2007).

47. Liu, H. et al. Capturing metastable structures during high-rate cycling of $\mathrm{LiFePO}_{4}$ nanoparticle electrodes. Science 344, 1252817 (2014).

48. Hardwick, L. J. et al. In situ Raman spectroscopy of insertion electrodes for lithium-ion batteries and supercapacitors: First cycle effects. J. Phys. Chem. Solids 69, 1232-1237 (2008).

49. Kilaas, R. Optimal and near-optimal filters in high-resolution electron microscopy. J. Microsc. 190, 45 (1998).

50. Wang, L., Zhou, F., Meng, Y. S. \& Ceder, G. First-principles study of surface properties of $\mathrm{Li} \mathrm{FePO}_{4}$ : surface energy, structure, wulff shape, and surface redox potential. Phys. Rev. B 76, 165435 (2007).

51. Karim, A., Fosse, S. \& Persson, K. A. Surface structure and equilibrium particle shape of the $\mathrm{LiMn}_{2} \mathrm{O}_{4}$ spinel from first-principles calculations. Phys. Rev. B 87, 075322 (2013).

52. Xiao, X. et al. Facile shape control of $\mathrm{Co}_{3} \mathrm{O}_{4}$ and the effect of the crystal plane on electrochemical performance. Adv. Mater. 24, 5762-5766 (2012).

53. Thackeray, M. M., Baker, S. D., Adendorff, K. T. \& Goodenough, J. B. Lithium insertion into $\mathrm{Co}_{3} \mathrm{O}_{4}$ : a preliminary investigation. Solid State Ion. 17, 175-181 (1985).

54. Porter, D. A. \& Easterling, K. E. Phase Transformations in Metals and Alloys 2nd edn (CRC, Boca Raton, FL, 2004).

55. Zhao, K. et al. Concurrent reaction and plasticity during initial lithiation of crystalline silicon in lithium-ion batteries. J. Electrochem. Soc. 159, A238-A243 (2012).

\section{Acknowledgements}

This work was supported as part of the Center for Mesoscale Transport Properties, an Energy Frontier Research Center supported by the U.S. Department of Energy (DOE), Office of Science, Basic Energy Sciences, under award \#DE SC0012673. This research used resources of the Center for Functional Nanomaterials, which is a U.S. DOE Office of Science Facility, at Brookhaven National Laboratory under contract No. DE-SC0012704. Q.M., L.W., and Y. Zhu are supported by U.S. DOE, DOE Office of Basic Energy Science, Division of Materials Science and Engineering, under Contract No. DE-SC0012704.

\section{Author contributions}

J.L., D.S. and E.A.S. designed the experiments. J.L. performed the in situ and ex situ TEM experiments. Y. Zhang, L.P., G.Y., K.J.T., A.C.M., and E.S.T. developed the synthesis approach, synthesized the large and small $\mathrm{ZnFe}_{2} \mathrm{O}_{4}$ samples. J.L. carried out the electrochemical tests. Q.M. performed the calculation. L.W. and Y. Zhu gave valuable comments on the crystallographic structure. J.L. prepared the figures and wrote the manuscript with E.A.S. All the authors participated in the discussion of the results and revision of the manuscript.

\section{Additional information}

Supplementary Information accompanies this paper at https://doi.org/10.1038/s41467018-07831-5.

Competing interests: The authors declare no competing interests.

Reprints and permission information is available online at http://npg.nature.com/ reprintsandpermissions/

Publisher's note: Springer Nature remains neutral with regard to jurisdictional claims in published maps and institutional affiliations.

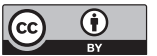

Open Access This article is licensed under a Creative Commons Attribution 4.0 International License, which permits use, sharing, adaptation, distribution and reproduction in any medium or format, as long as you give appropriate credit to the original author(s) and the source, provide a link to the Creative Commons license, and indicate if changes were made. The images or other third party material in this article are included in the article's Creative Commons license, unless indicated otherwise in a credit line to the material. If material is not included in the article's Creative Commons license and your intended use is not permitted by statutory regulation or exceeds the permitted use, you will need to obtain permission directly from the copyright holder. To view a copy of this license, visit http://creativecommons.org/ licenses/by/4.0/.

(c) This is a U.S. Government work and not under copyright protection in the US; foreign copyright protection may apply 2019 